\title{
AFS Theory based Integrated Multiple Criteria Decision Making Model for Transportation Mode Selection
}

\author{
Sunil Pratap Singh ${ }^{1 *}$ and Preetvanti Singh ${ }^{2}$ \\ ${ }^{1}$ Bharati Vidyapeeth's Institute of Computer Applications and Management, India \\ ${ }^{2}$ Dayalbagh Educational Institute (Deemed University), India \\ ${ }^{1}$ sunil_pratap@rediffmail.com, ${ }^{2}$ preetvantisingh@gmail.com
}

\begin{abstract}
This paper presents the development of a novel hybrid decision support model aimed to solve the complex problem of transportation mode selection in multiple criteria decision making environment. The proposed model integrates Analytic Hierarchy Process (AHP) to determine relative importance of transportation mode selection attributes; Axiomatic Fuzzy Set (AFS) theory for description of decision alternatives; and Simple Additive Weighting $(S A W)$ technique to obtain the final ranking of alternatives. The axiomatic fuzzy logic is incorporated into the model to overcome the uncertainty and ambiguity in human decision making process. The main advantage of the proposed model is that it processes the linguistic values using axiomatic fuzzy logic that overcomes the ambiguity in human decision making process and copes with the inconsistency caused by different types of fuzzy numbers and normalization methods. The proposed methodology is illustrated by considering a case of transportation mode selection. A comparative analysis with an established technique shows the effectiveness and validity of the proposed model.
\end{abstract}

Keywords: Hybrid Method, Transportation Mode Selection, Travel Mode, Multiple Criteria Decision Making, Axiomatic Fuzzy Set (AFS) Theory, Simple Additive Weighting $(S A W)$

\section{Introduction}

In everyday life, people face decision situations in their professional as well as private lives. In general, the decision-making process involves a sequence of logical steps to resolve a complex problem. The process begins with recognizing the decision problem and ends with determining a best alternative from among multiple alternatives for the purpose of attaining a goal or goals [6].

The multiple criteria decision making (MCDM) is one of the most well-known branch of decision making that deals with the complex problems under the presence of a set of decision criteria. The term MADM (multiple attribute decision making) is used interchangeably with MCDM. The development of MCDM techniques and their applications in solving complex decision problems have shown a remarkable growth in last few years [3].

Generally, a MCDM problem can be concisely expressed in matrix form as:

Received (May 25, 2018), Review Result (July 10, 2018), Accepted (July 13, 2018)

* Corresponding Author 


\begin{tabular}{l|llll} 
& \multicolumn{1}{l}{$C_{1}$} & $C_{2}$ & $\ldots$ & \multicolumn{1}{c}{$C_{n}$} \\
$A_{1}$ & $a_{11}$ & $a_{12}$ & $\ldots$ & $a_{1 n}$ \\
$A_{2}$ & $a_{21}$ & $a_{22}$ & $\ldots$ & $a_{2 n}$ \\
$\vdots$ & $\vdots$ & $\vdots$ & $\ddots$ & $\vdots$ \\
$A_{m}$ & $a_{m 1}$ & $a_{m 2}$ & $\ldots$ & $a_{m n}$ \\
$W=\left[w_{1}, w_{2}, \ldots, w_{n}\right]$
\end{tabular}

where $A=\left\{A_{i} \mid i=1,2, \ldots, m\right\}$ is a set of possible alternatives among which decision makers have to choose; $C=\left\{C_{j} \mid j=1,2, \ldots, n\right\}$ is a set of criteria with which alternative's performances are measured, $a_{i j}, i=1, \ldots, m ; j=1, \ldots, n$ is the rating of alternative $A_{i}$ with respect to criterion $C_{j}, j=1, \ldots, n$ and $w_{j}$ is the weight of criterion $C_{j}$.

In practice, it is common to find complex decision-making situations involving both, quantitative and qualitative aspects. The quantitative aspects are generally assessed by means of precise numerical values, but qualitative aspects are complex to assess with precise and exact values. It is not possible to model such imprecise situations using traditional MCDM approaches and requires combining these with fuzzy logic and other techniques in a hybrid manner to deal with the qualitative aspects and uncertainty in decision-making process [13]. However, the fuzzy set theory deals with fuzzy numbers and the use of these different fuzzy numbers given by decision makers in different knowledge areas lead to different results. It may also be difficult to select an appropriate method for normalising the decision matrix (converting the criteria values into the dimensionless form) since a lot of normalisation methods have been developed and the choice of different methods might change the final selection and ranking for a specific problem [1].

The main objective of this research study is to develop a hybrid decision support model, for assisting the travellers in intelligent decision-making activities, by combining multicriteria decision-making and fuzzy logic techniques. The AFS theory is incorporated into the MCDM techniques to model fuzziness in human knowledge representation and reasoning process. The developed hybrid model integrates AHP, SAW and AFS theory to contribute a new way for solving complex problems under MCDM environment. It takes advantages of normalisation of decision matrix under the AHP calculation framework and inconsistency caused by different types of fuzzy numbers.

The presentation of our work on developing the hybrid decision support model in this paper is organised as follows. In Section 2, the background of MCDM techniques (AHP and SAW) is provided. The concept of AFS theory is also discussed in Section 2. Section 3 outlines the methodological steps of the proposed model. Section 4 presents the utilisation of developed decision support model in a real life example of transportation mode selection. In Section 5, a comparative analysis and validation is reported, and finally, Section 6 concludes the paper.

\section{Theoretical Background}

Although the theoretical backgrounds of AHP, AFS theory, and SAW have been documented in the literature, a brief outline of these methods is included below in the context of the present work.

\subsection{Analytic Hierarchy Process (AHP)}

AHP, developed by Saaty [19], is a mathematical technique which addresses how to determine the relative importance of a set of criteria in a decision problem. The ability to incorporate judgments on intangible qualitative criteria alongside tangible quantitative 
criteria makes AHP an ideal methodology for prioritisation problems having a set of potentially conflicting criteria. The literature on AHP applications is very rich [10]. In recent research, AHP has been applied to solve many complex decision problems [11], [2], [18], [17] [5].

The process of AHP can be summarised in four steps: construct the decision hierarchy, determine the relative importance of criteria and sub-criteria, evaluate each alternative and calculate its overall weight in regard to each criterion, and check the consistency of the subjective evaluations [19]. In the first step, the problem is organised as a hierarchical structure of decision elements where the top level represents the goal of decision, second and subsequent levels represent criteria and sub-criteria, and the lowest level represents the decision alternatives. In the second step, the decision maker is asked to subjectively evaluate pairs of criteria based on Saaty's [19] standardised scale of nine levels, as given in Table 1. In the third step, a relative importance value (weight) is computed for each criterion (and sub-criterion) based on pairwise comparisons. The logical consistency in comparison process is tested in the last step.

Table 1. Scale for Pairwise Comparison (Saaty [19])

\begin{tabular}{|c|c|c|c|c|c|c|}
\hline $\begin{array}{c}\text { Intensity of } \\
\text { Importance }\end{array}$ & 1 & 3 & 5 & 7 & 9 & $2,4,6,8$ \\
\hline Definition & Equal & Moderate & Strong & Demonstrated & Extreme & Intermediate Value \\
\hline
\end{tabular}

If we wish to compare a set of $n$ criteria pairwise according to their relative importance, then the result of pairwise comparisons can be summarised in an $(n \times n)$ algebraic matrix $P$, as shown:

$$
P=\left[\begin{array}{cccc}
p_{11} & p_{12} & \ldots & p_{1 n} \\
p_{21} & p_{22} & \ldots & p_{2 n} \\
\vdots & \vdots & \ddots & \vdots \\
p_{n 1} & p_{n 2} & \ldots & p_{n n}
\end{array}\right]
$$

where $p_{i j}$ is the relative importance for $i$ to $j, p_{j i}=1 / p_{i j}$ and $p_{i j}=1$ if $i=j$.

The process commences to normalise the pairwise comparison matrix and obtain the relative weights. The relative weights are given by right eigenvector $(w)$ corresponding to the largest eigenvalue $\left(\lambda_{\max }\right)$, as:

$$
P w=\lambda_{\max } w
$$

In order to ensure the consistency of subjective perception in pairwise comparison, two indices, consistency index (CI) and consistency ratio (CR) are suggested. The consistency index for the matrix of order $n$ is expressed as:

$$
\mathrm{CI}=\frac{\left(\lambda_{\max }-n\right)}{(n-1)}
$$

The $\mathrm{CR}$ is calculated as the ratio of $\mathrm{CI}$ and random index (RI), as indicated:

$$
\mathrm{CR}=\frac{\mathrm{CI}}{\mathrm{RI}}
$$

where RI refers to a random consistency index derived from a randomly generated pairwise comparison matrix. The random indices with respect to different size matrices are shown in Table 2. If the calculated CR of a pairwise comparison is less than 0.1 , the decision maker's judgment is consistent and acceptable; otherwise the evaluation procedure is considered inconsistent and needs revision to improve consistency. 
Table 2. Random Consistency Indices (Saaty [19])

\begin{tabular}{|c|c|c|c|c|c|c|c|c|c|c|}
\hline$n$ & 1 & 2 & 3 & 4 & 5 & 6 & 7 & 8 & 9 & 10 \\
\hline $\mathrm{RI}$ & 0.00 & 0.00 & 0.58 & 0.90 & 1.12 & 1.24 & 1.32 & 1.41 & 1.46 & 1.49 \\
\hline
\end{tabular}

\subsection{Axiomatic Fuzzy Set (AFS) Theory}

In everyday life, we come across complex decision situations, the nature of which is ambiguous and imprecise. It is not possible to formally describe such imprecise situations using classical set theory and bivalent logic. The AFS theory, proposed by Liu [23], is a mathematical framework that aims to explore how fuzzy set theory and probability can be made to work in concert, so that the uncertainty of randomness and of imprecision can be treated in a unified and coherent manner. It provides an effective tool to convert the information in observed data into the membership functions and logic operations of fuzzy concepts. The AFS theory is based on the AFS algebra - a kind of semantic methodology of fuzzy concepts and AFS structure - a kind of mathematical description of data structures. The recent literature of AFS studies and their applications ([27], [28], [24], [25], [14], [17], [26]) reveals that it is a flexible and powerful framework for representing human knowledge and studying intelligent systems in real world applications.

2.2.1. AFS Algebra: Liu [23] defined a family of completely distributive lattices, referred to as AFS algebras and applied them to study the semantics of expressions and representations of fuzzy concepts. In a multiple criteria decision problem, let $X=$ $\left\{x_{1}, x_{2}, \ldots, x_{5}\right\}$ be a set of alternatives, $M=\left\{m_{1}, m_{1}^{\prime}, \ldots, m_{5}, m_{5}^{\prime}\right\}$ be a set of fuzzy attributes on $X$, where $m_{1}$ : "attribute 1 is good", $m_{1}^{\prime}$ : "attribute 1 is not good", .., $m_{5}$ : "attribute 5 is good", $m_{5}^{\prime}$ : "attribute 5 is not good". For each set of concepts $A \subseteq$ $M, \prod_{m \in A} m$ represents conjunction of the concepts in $A . \sum_{i \in I}\left(\prod_{m \in A_{i}} m\right)$, a formal sum of $\prod_{m \in A_{i}} m, A_{i} \subseteq M, i \in I$, is the disjunction of the conjunctions represented by $\prod_{m \in A_{i}} m$ 's (i.e., the disjunctive normal form of a formula representing a concept). For instance, we may have $m_{1} m_{5}+m_{1} m_{3}+m_{4}$ which translates as "attribute 1 and attribute 5 are good" or "attribute 1 and attribute 3 are good" or "attribute 4 is good" (the "+" sign represents disjunction of concepts). For $A_{i} \subseteq M, i \in I, \sum_{i \in I}\left(\prod_{m \in A_{i}} m\right)$ has a well-defined meaning as discussed above. The semantics of the logic expressions such as "equivalent to", "or", and "and", as expressed by $\sum_{i \in I}\left(\prod_{m \in A_{i}} m\right)$, can be formulated in terms of the AFS algebra $\left(E M^{*}\right)$, defined as:

$$
E M^{*}=\left\{\sum_{i \in I}\left(\prod_{m \in A_{i}}^{m}\right) \mid A_{i} \subseteq M, i \in I, I \text { is an non-empty indexing set }\right\}
$$

Definition 1 [23]: Let $M$ be a non-empty set, a binary relation $R$ on $E M^{*}$ is defined as follows:

$\forall \sum_{i \in I}\left(\prod_{m \in A_{i}} m\right)$ and $\sum_{j \in J}\left(\prod_{m \in B_{j}} m\right) \in E M^{*}$,

$\left[\sum_{i \in I}\left(\prod_{m \in A_{i}} m\right) R \sum_{j \in J}\left(\prod_{m \in B_{j}} m\right)\right] \Leftrightarrow(\mathrm{i}) \forall A_{i}(i \in I), \exists B_{h}(h \in J)$ such that $B_{h} \subseteq$ $A_{i}$, and (ii) $\forall B_{j}(j \in J), \exists A_{k}(k \in I)$ such that $A_{k} \subseteq B_{j}$.

It is obvious that $R$ is an equivalence relation and the quotient set $\left(E M^{*} / R\right)$ is denoted by $E M$. The notation $\sum_{i \in I}\left(\prod_{m \in A_{i}} m\right)=\sum_{j \in J}\left(\prod_{m \in B_{j}} m\right)$ means that $\sum_{i \in I}\left(\prod_{m \in A_{i}} m\right)$ and $\sum_{j \in J}\left(\prod_{m \in B_{j}} m\right)$ are equivalent (represents same semantics) under relation $R$.

Theorem 1 [23]: Let $M$ be a non-empty set, then $(E M, \wedge, \vee)$ forms a completely distributive lattice under the binary compositions $\wedge$ and $\vee$, defined as follows: 


$$
\begin{aligned}
& \forall \sum_{i \in I}\left(\prod_{m \in A_{i}} m\right), \sum_{j \in J}\left(\prod_{m \in B_{j}} m\right) \in E M^{*}, \\
& \sum_{i \in I}\left(\prod_{m \in A_{i}} m\right) \wedge \sum_{j \in J}\left(\prod_{m \in B_{j}} m\right)=\sum_{i \in I, j \in J}\left(\prod_{m \in A_{i} \cup B_{j}} m\right) \\
& \sum_{i \in I}\left(\prod_{m \in A_{i}} m\right) \vee \sum_{j \in J}\left(\prod_{m \in B_{j}} m\right)=\sum_{k \in I \sqcup J}\left(\prod_{m \in C_{k}} m\right)
\end{aligned}
$$

where for any $k \in I \sqcup J$ (the disjoint union of $I$ and $J$, i.e., every element in $I$ and every element in $J$ are always regarded as different elements in $I \sqcup J) ; C_{k}=A_{k}$ if $k \in I$ and $C_{k}=B_{k}$ if $k \in J .(E M, \wedge, \mathrm{V})$ is called the $E I$ (expending on $M$ ) algebra over $M$.

2.2.2. AFS Structure: An AFS structure represented by a triple $(M, \tau, X)$, gives rise to membership functions and fuzzy logic operations of the concepts in $E M$.

Definition 2 [22], [23]: Let $X, M$ be sets and $\tau: X \times X \rightarrow 2^{M}$. $(M, \tau, X)$ is called an AFS structure if $\tau$ satisfies the following axioms:

$$
\begin{aligned}
& \text { (a) }) . \forall\left(x_{1}, x_{2}\right) \in X \times X, \tau\left(x_{1}, x_{2}\right) \subseteq \tau\left(x_{1}, x_{1}\right) \\
& \text { (a) }) . \forall\left(x_{1}, x_{2}\right),\left(x_{2}, x_{3}\right) \in X \times X, \tau\left(x_{1}, x_{2}\right) \cap \tau\left(x_{2}, x_{3}\right) \subseteq \tau\left(x_{1}, x_{3}\right)
\end{aligned}
$$

$X$ is called the universe of discourse, $M$ is called the concept set and $\tau$ is called the structure. In real world applications, $\tau$ can be constructed from a linearly ordered relation $\left(\geq_{m}\right)$ as follows:

$$
\tau(x, y)=\left\{m \mid m \in M, x \geq_{m} y\right\} \subseteq 2^{M}
$$

where , $x \geq_{m} y$ implies that the degree of $x$ belonging to simple concept $m$ is greater than or equal to $y$.

Definition 3 [22]: Let $X$ and $M$ be sets, $(M, \tau, X)$ be an AFS structure and $(M, \sigma, m)$ be a measure space, where $m$ is a finite and positive measure, $m(X) \neq 0, A_{i}^{\tau} \in \sigma, x \in X, i \in$ $I$. For the fuzzy concept $\eta=\sum_{i \in I}\left(\prod_{m \in A_{i}} m\right) \in E M$, the membership function of $\eta$ is defined as follows:

For any $x \in X$,

$$
\mu_{\eta}(x)=\sup _{i \in I} \frac{m\left(A_{i}^{\tau}(x)\right)}{m(X)}
$$

where $A_{i}^{\tau}(x)=\left\{y \in X: x \geq_{m} y\right.$, for any $\left.m \in A_{i}\right\}$. In other words, $A_{i}^{\tau}$ is the set of all elements in $X$ whose degree of belonging to concept $\prod_{m \in A_{i}} m$ are less than or equal to that of $x$.

\subsection{Simple Additive Weighting (SAW)}

The simple additive weighting (SAW), also known as weighted sum model, is one of the most popular and simple multicriteria decision analysis technique for evaluating a set of alternatives in terms of a set of criteria. In a multiple criteria decision problem with $m$ alternatives and $n$ criteria, the performance score of each alternative can be derived using following equation:

$$
A_{i}^{*}=\sum_{j=1}^{n} a_{i j} w_{j}
$$

where $a_{i j}$ (for $i=1,2, \ldots, m$ and $j=1,2, \ldots, n$ ) represents the normalized rating of alternative $i$ with respect to criterion $j$ and $w_{j}$ represents the normalized weight of criterion $j$. The alternative with maximum performance score $\left(A_{i}^{*}\right)$ is considered as best alternative. 


\section{Proposed Model: Hybrid Decision Support Model}

The subjective and qualitative nature of a multiple criteria decision-making problem requires expressing the strength or weakness of preferences using linguistic terms. In practice, the linguistic values are represented by different types of fuzzy numbers (triangular fuzzy number, trapezoidal fuzzy number, etc.,) which may lead to different results. The proposed methodological framework utilizes AFS theory in AHP and SAW to develop a hybrid model to solve complex MCDM problems. The linguistic values are processed with AFS theory to cope with the inconsistency caused by different types of fuzzy numbers. The hybrid model consists of five basic stages:

Stage 1: Identification of decision criteria and linguistic assessment of alternatives with respect to criteria using modified-delphi method.

Step 1: Identify all possible decision alternatives: $A_{i}(i=1, \ldots, m)$ and criteria: $C_{j}(j=$ $1, \ldots, n)$.

Step 2: Establish a judgment matrix $\left[a_{i j}\right]_{m \times n}$ for linguistic assessment of alternatives in terms of decision criteria. The matrix element $a_{i j}$ represents assessment of $i^{\text {th }}$ alternative in terms of $j^{\text {th }}$ criteria using linguistic terms.

Stage 2: Computation of relative weights $\left(w_{j}\right)$ of decision criteria.

Step 1: Establish a comparison matrix $\left[p_{i j}\right]_{n \times n}$ by performing pairwise comparisons (using 9-point scale) between each pair of criteria.

Step 2: Compute the weights of criteria $\left(w_{j}\right)$ using AHP.

Stage 3: Fuzzy description $\left(\zeta_{A_{i}}\right)$ of each decision alternative using AFS theory. According to Liu and Pedrycz [21], the fuzzy description for each alternative is determined by the following steps:

Step 1: Find the set of fuzzy attributes in $M$, defined as:

$$
B_{A_{i}}^{\varepsilon}=\left\{m_{k} \in M: \mu_{m_{k}}\left(A_{i}\right) \geq \mu_{v}\left(A_{i}\right)-\epsilon\right\}
$$

Step 2: Find the set $\bar{B}_{A_{i}}^{\varepsilon}$, defined as follows:

$$
\bar{B}_{A_{i}}^{\varepsilon}=\left\{\prod_{m \in A} m: \mu_{\prod_{m \in A} m}\left(A_{i}\right) \geq \mu_{v}\left(A_{i}\right)-\varepsilon, A \subseteq B_{A_{i}}^{\varepsilon}\right\}
$$

Step 3: Select the best fuzzy description $\zeta_{A_{i}} \in \bar{B}_{A_{i}}^{\varepsilon}$ for the alternative $A_{i}$ as follows:

$$
\zeta_{A_{i}}=\arg \min _{\zeta \in \bar{B}_{A_{i}}^{\varepsilon}}\left\{\sum_{x \in X, x \neq A_{i}} \mu_{\zeta}(x)\right\}
$$

Stage 4: Establish a decision matrix by rating each alternative $A_{i}(i=1,2, \ldots, m)$ over each decision criterion $C_{j}(j=1,2 \ldots, n)$.

Step 1: Perform pairwise comparisons (using 9-point scale) between each pair of decision alternatives according to benefit and cost criterion in their best fuzzy descriptions $\left(\zeta_{A_{i}}\right)$. For benefit criterion, the alternatives are compared based on $C_{j}$ (criterion $C_{j}$ is good) and for cost criterion, the comparison process is carried out based on $C_{j}^{\prime}$ (criterion $C_{j}$ is not good).

Step 2: Compute the performance scores of alternatives over decision criteria using AHP and establish a normalised decision matrix where each element $\left(r_{i j}\right)$ represents the 
performance score of $i^{\text {th }}$ alternative over $j^{\text {th }}$ criteria. Since the performance scores $\left(r_{i j}\right)$ are obtained using AHP, these are considered as normalised under AHP calculation framework and therefore there is no need to further normalise them explicitly.

Stage 5: Ranking the decision alternatives.

Step 1: Establish a weighted normalised decision matrix and evaluate the performance of each alternative using Equation (12) and rank accordingly.

\section{Application of Proposed Model in Transportation Mode Selection}

The proposed model is applied to a real world application of transportation mode selection in Agra, a historic city in northern region of India. The city has an international importance in tourism and industrial sector which makes it flooded with naïve commuters. The selection of best mode of transportation in advance may help the travelers in meeting their personalized preferences and making journey efficient. In following section, the application of proposed decision support model is described in reference to selecting best mode of transportation based on personalized preferences. There are various modes of transportation in Agra, this study considers only six most commonly used modes of transportation - $A_{1}$ : auto rickshaw, $A_{2}$ : cycle rickshaw, $A_{3}$ : electric rickshaw, $A_{4}$ : taxi, $A_{5}$ : bus, and $A_{6}$ : train. The following section illustrates the step by step process of the developed hybrid model in evaluating best means of transportation based on a set of decision criteria.

Stage 1: Identification of decision criteria and linguistic assessment of alternatives with respect to criteria using modified-Delphi method.

This phase begins with forming a team of 5 experts responsible for identifying a set of selection criteria important to determine best mode of transportation. A list of selection criteria was prepared based on expert's opinion and literature survey ([20], [15], [12], [4], [16], [8], [7]) and a calibration process was applied to narrow down the list to include only those criteria the travelers' feel pertinent to select a transportation mode. This process resulted in a set of eight criteria $-C_{1}$ : safety, $C_{2}$ : speed, $C_{3}$ : travel cost, $C_{4}$ : interchange, $C_{5}$ : accessibility, $C_{6}$ : comfort, $C_{7}$ : environment friendliness, and $C_{8}$ : capacity. Next, the expert's perceptions (linguistic assessment) of transportation modes with respect to selection criteria are elicited. Table 3 shows the result of the elicitation process.

Table 3. Linguistic Assessment of Transportation Modes

\begin{tabular}{|c|c|c|c|c|c|c|c|c|}
\hline \multirow{2}{*}{ Modes } & \multicolumn{9}{|c|}{ Decision Criteria } \\
\cline { 2 - 9 } & $C_{1}$ & $C_{2}$ & $C_{3}$ & $C_{4}$ & $C_{5}$ & $C_{6}$ & $C_{7}$ & $C_{8}$ \\
\hline$A_{1}$ & $\mathrm{H}$ & $\mathrm{H}$ & $\mathrm{H}$ & $\mathrm{L}$ & $\mathrm{VH}$ & $\mathrm{H}$ & $\mathrm{ML}$ & $\mathrm{ML}$ \\
\hline$A_{2}$ & $\mathrm{ML}$ & $\mathrm{L}$ & $\mathrm{MH}$ & $\mathrm{MH}$ & $\mathrm{VH}$ & $\mathrm{ML}$ & $\mathrm{H}$ & $\mathrm{L}$ \\
\hline$A_{3}$ & $\mathrm{~L}$ & $\mathrm{MH}$ & $\mathrm{MH}$ & $\mathrm{MH}$ & $\mathrm{H}$ & $\mathrm{ML}$ & $\mathrm{H}$ & $\mathrm{ML}$ \\
\hline$A_{4}$ & $\mathrm{H}$ & $\mathrm{H}$ & $\mathrm{VH}$ & $\mathrm{VL}$ & $\mathrm{MH}$ & $\mathrm{VH}$ & $\mathrm{MH}$ & $\mathrm{MH}$ \\
\hline$A_{5}$ & $\mathrm{MH}$ & $\mathrm{MH}$ & $\mathrm{ML}$ & $\mathrm{VH}$ & $\mathrm{ML}$ & $\mathrm{MH}$ & $\mathrm{L}$ & $\mathrm{H}$ \\
\hline$A_{6}$ & $\mathrm{MH}$ & $\mathrm{MH}$ & $\mathrm{L}$ & $\mathrm{VH}$ & $\mathrm{MH}$ & $\mathrm{MH}$ & $\mathrm{ML}$ & $\mathrm{VH}$ \\
\hline
\end{tabular}

The meaning of linguistic ratings of different criteria in Table 3 can be defined as: VL $=$ Very Low, $\mathrm{L}=$ Low, $\mathrm{ML}=$ Medium Low, $\mathrm{M}=$ Medium, $\mathrm{MH}=$ Medium High, $\mathrm{H}=$ High, VH = Very High.

Stage 2: Computation of relative weights $\left(w_{j}\right)$ of decision criteria. 
In this phase, a pairwise comparison matrix $P=\left(p_{i j}\right)_{n \times n}$ is established based on traveler's preferences to decision criteria (see Table 4) which results in computation of criteria weights $\left(w_{j}\right)$ using eigenvalue calculation framework.

Stage 3: Fuzzy description $\left(\zeta_{A_{i}}\right)$ of each decision alternative using AFS theory.

Let $X=\left\{A_{1}, A_{2}, A_{3}, A_{4}, A_{5}, A_{6}\right\}$ be the set of six transportation modes, $\epsilon=0, C=$ $\left\{C_{1}, C_{1}^{\prime}, C_{2}, C_{2}^{\prime}, \ldots, C_{8}, C_{8}^{\prime}\right\}$ be the set of decision criteria on $X$ and $v=C_{1}+C_{1}^{\prime}+C_{2}+C_{2}^{\prime}+$ $\cdots C_{8}+C_{8}^{\prime}$. By Table 3 and semantic meaning of decision criteria, we have following linearly ordered relations:

$$
\begin{array}{ll}
C_{1}: A_{3}<A_{2}<A_{5}=A_{6}<A_{1}=A_{4} & C_{1}^{\prime}: A_{3}>A_{2}>A_{5}=A_{6}>A_{1}=A_{4} \\
C_{2}: A_{2}<A_{3}=A_{5}=A_{6}<A_{1}=A_{4} & C_{2}^{\prime}: A_{2}>A_{3}=A_{5}=A_{6}>A_{1}=A_{4} \\
C_{3}: A_{6}<A_{5}<A_{2}=A_{3}<A_{1}<A_{4} & C_{3}^{\prime}: A_{6}>A_{5}>A_{2}=A_{3}>A_{1}>A_{4} \\
C_{4}: A_{4}<A_{1}<A_{2}=A_{3}<A_{5}=A_{6} & C_{4}^{\prime}: A_{4}>A_{1}>A_{2}=A_{3}>A_{5}=A_{6} \\
C_{5}: A_{5}<A_{4}=A_{6}<A_{3}<A_{1}=A_{2} & C_{5}^{\prime}: A_{5}>A_{4}=A_{6}>A_{3}>A_{1}=A_{2} \\
C_{6}: A_{2}=A_{3}<A_{5}=A_{6}<A_{1}<A_{4} & C_{6}^{\prime}: A_{2}=A_{3}>A_{5}=A_{6}>A_{1}>A_{4} \\
C_{7}: A_{5}<A_{1}=A_{6}<A_{4}<A_{2}=A_{3} & C_{7}^{\prime}: A_{5}>A_{1}=A_{6}>A_{4}>A_{2}=A_{3} \\
C_{8}: A_{2}<A_{1}=A_{3}<A_{4}<A_{5}<A_{6} & C_{8}^{\prime}: A_{2}>A_{1}=A_{3}>A_{4}>A_{5}>A_{6}
\end{array}
$$

To distinguish $A_{i}$ from other modes of transportation in $X$, the best fuzzy description of each mode $\left(\zeta_{A_{i}}\right)$ is obtained as follows:

By Equation (11),

$$
\mu_{v}\left(A_{1}\right)=\mu_{v}\left(A_{2}\right)=\mu_{v}\left(A_{3}\right)=\mu_{v}\left(A_{4}\right)=\mu_{v}\left(A_{5}\right)=\mu_{v}\left(A_{6}\right)=1.0
$$

By Equation (11), (13), (14), and (15),

$\mu_{C_{1}}\left(A_{1}\right)=\mu_{C_{2}}\left(A_{1}\right)=\mu_{C_{5}}\left(A_{1}\right)=1.0$

$B_{A_{1}}^{0}=\left\{C_{1}, C_{2}, C_{5}\right\}$ and $\mu_{C_{1} C_{2} C_{5}}$ is the minimal element in $\bar{B}_{A_{1}}^{\varepsilon}$.

$\zeta_{A_{1}}=C_{1} C_{2} C_{5}$

The fuzzy description $\zeta_{A_{1}}$ can be interpreted as: "the transport mode $A_{1}$ has good safety, speed and accessibility".

Similarly,

$$
\begin{aligned}
& B_{A_{2}}^{0}=\left\{C_{5}, C_{7}, C_{2}^{\prime}, C_{6}^{\prime}, C_{8}^{\prime}\right\} \Rightarrow \zeta_{A_{2}}=C_{5} C_{7} C_{2}^{\prime} C_{6}^{\prime} C_{8}^{\prime} \\
& B_{A_{3}}^{0}=\left\{C_{7}, C_{1}^{\prime}, C_{6}^{\prime}\right\} \Rightarrow \zeta_{A_{3}}=C_{7} C_{1}^{\prime} C_{6}^{\prime} \\
& B_{A_{4}}^{0}=\left\{C_{1}, C_{2}, C_{3}, C_{6}, C_{4}^{\prime}\right\} \Rightarrow \zeta_{A_{4}}=C_{1} C_{2} C_{3} C_{6} C_{4}^{\prime} \\
& B_{A_{5}}^{0}=\left\{C_{4}, C_{5}^{\prime}, C_{7}^{\prime}\right\} \Rightarrow \zeta_{A_{5}}=C_{4} C_{5}^{\prime} C_{7}^{\prime} \\
& B_{A_{6}}^{0}=\left\{C_{4}, C_{8}, C_{3}^{\prime}\right\} \Rightarrow \zeta_{A_{6}}=C_{4} C_{8} C_{3}^{\prime}
\end{aligned}
$$


Table 4. Pairwise Comparison Matrix and Criteria Weights

\begin{tabular}{|c|c|c|c|c|c|c|c|c|}
\hline Criteria & $C_{1}$ & $C_{2}$ & $C_{3}$ & $C_{4}$ & $C_{5}$ & $C_{6}$ & $C_{7}$ & $C_{8}$ \\
\hline$C_{1}$ & 1.000 & 3.000 & 2.000 & 3.000 & 2.000 & 2.000 & 3.000 & 1.000 \\
\hline$C_{2}$ & & 1.000 & 0.500 & 2.000 & 0.500 & 0.500 & 2.000 & 0.500 \\
\hline$C_{3}$ & & & 1.000 & 2.000 & 1.000 & 1.000 & 3.000 & 2.000 \\
\hline$C_{4}$ & & & & 1.000 & 0.500 & 0.500 & 1.000 & 0.333 \\
\hline$C_{5}$ & & & & & 1.000 & 1.000 & 0.500 & 2.000 \\
\hline$C_{6}$ & & & & & & 1.000 & 2.000 & 2.000 \\
\hline$C_{7}$ & & & & & & & 1.000 & 0.500 \\
\hline$C_{8}$ & & & & & & & & 1.000 \\
\hline$w_{j}$ & 0.234 & 0.084 & 0.153 & 0.060 & 0.129 & 0.144 & 0.082 & 0.113 \\
\hline
\end{tabular}

Stage 4: Establish a decision matrix by rating each alternative $A_{i}(i=1,2, \ldots, m)$ over each decision criterion $C_{j}(j=1,2 \ldots, n)$.

This phase starts with rating the transportation modes according to their fuzzy description. In this process, the transportation modes are rated over each criterion by considering $C_{1}, C_{2}, C_{5}, C_{6}, C_{7}, C_{8}$ as the benefit criteria and $C_{3}, C_{4}$ as the cost criteria. For illustration, the rating of all transportation modes over the criterion $C_{1}$ is presented in Table 5 with explanation as - since $C_{1}$ appears in $\zeta_{A_{1}}$ and $\zeta_{A_{4}}$, hence $A_{1}$ and $A_{4}$ are extremely preferred over $A_{2}, A_{3}, A_{5}$ and $A_{6}$. Similarly, the weights of all transportation modes over each criterion $C_{j}: j=1,2, \ldots, 8$ are obtained and a normalised (since the weights determined using AHP are already normalised, therefore no further normalisation is required) fuzzy decision matrix is obtained in Table 6.

Table 5. Rating of Transportation Modes over Criterion $C_{1}$

\begin{tabular}{|c|c|c|c|c|c|c|c|}
\hline $\boldsymbol{C}_{\boldsymbol{1}}$ & $A_{1}$ & $A_{2}$ & $A_{3}$ & $A_{4}$ & $A_{5}$ & $A_{6}$ & Weight \\
\hline$A_{1}$ & 1 & 9 & 1 & 1 & 9 & 9 & 0.409 \\
\hline$A_{2}$ & $1 / 9$ & 1 & $1 / 9$ & $1 / 9$ & 1 & 1 & 0.045 \\
\hline$A_{3}$ & 1 & 9 & 1 & 1 & 9 & 9 & 0.045 \\
\hline$A_{4}$ & 1 & 9 & 1 & 1 & 9 & 9 & 0.409 \\
\hline$A_{5}$ & $1 / 9$ & 1 & $1 / 9$ & $1 / 9$ & 1 & 1 & 0.045 \\
\hline$A_{6}$ & $1 / 9$ & 1 & $1 / 9$ & $1 / 9$ & 1 & 1 & 0.045 \\
\hline
\end{tabular}

Table 6. Normalised Decision Matrix

\begin{tabular}{|c|c|c|c|c|c|c|c|c|}
\hline \multirow{2}{*}{ Modes } & \multicolumn{7}{|c|}{ Decision Criteria } \\
\cline { 2 - 9 } & $C_{1}$ & $C_{2}$ & $C_{3}^{\prime}$ & $C_{4}^{\prime}$ & $C_{5}$ & $C_{6}$ & $C_{7}$ & $C_{8}$ \\
\hline$A_{1}$ & 0.409 & 0.409 & 0.071 & 0.071 & 0.409 & 0.071 & 0.045 & 0.071 \\
\hline$A_{2}$ & 0.045 & 0.045 & 0.071 & 0.071 & 0.409 & 0.071 & 0.409 & 0.071 \\
\hline$A_{3}$ & 0.045 & 0.045 & 0.071 & 0.071 & 0.045 & 0.071 & 0.409 & 0.071 \\
\hline$A_{4}$ & 0.409 & 0.409 & 0.071 & 0.643 & 0.045 & 0.643 & 0.045 & 0.071 \\
\hline$A_{5}$ & 0.045 & 0.045 & 0.071 & 0.071 & 0.045 & 0.071 & 0.045 & 0.071 \\
\hline$A_{6}$ & 0.045 & 0.045 & 0.643 & 0.071 & 0.045 & 0.071 & 0.045 & 0.643 \\
\hline
\end{tabular}

In heading of Table $6, C_{3}^{\prime}$ and $C_{4}^{\prime}$ indicate that these are cost criteria for which the minimum value will be preferred and hence $A_{i}$ 's are rated over $C_{3}^{\prime}$ and $C_{4}^{\prime}$ in order to 
minimise them.

Stage 5: Ranking the decision alternatives.

In this last phase, a weighted normalised decision matrix is established (Table 7) and the performance of each transportation mode (rank) is evaluated using Equation (12). The graphical representation of overall performance scores of transportation modes is shown in Figure 1.

Table 7. Weighted Normalised Decision Matrix and Final Ranking based on SAW

\begin{tabular}{|c|c|c|c|c|c|c|c|c|c|c|}
\hline \multirow{2}{*}{ Modes } & \multicolumn{7}{|c|}{ Decision Criteria } & \\
\cline { 2 - 11 } & $C_{1}$ & $C_{2}$ & $C_{3}^{\prime}$ & $C_{4}^{\prime}$ & $C_{5}$ & $C_{6}$ & $C_{7}$ & $C_{8}$ & $A_{i}^{*}$ & Rank \\
\hline$A_{1}$ & 0.096 & 0.034 & 0.011 & 0.004 & 0.053 & 0.010 & 0.004 & 0.008 & 0.220 & 2 \\
\hline$A_{2}$ & 0.011 & 0.004 & 0.011 & 0.004 & 0.053 & 0.010 & 0.034 & 0.008 & 0.134 & 4 \\
\hline$A_{3}$ & 0.011 & 0.004 & 0.011 & 0.004 & 0.006 & 0.010 & 0.034 & 0.008 & 0.087 & 5 \\
\hline$A_{4}$ & 0.096 & 0.034 & 0.011 & 0.039 & 0.006 & 0.093 & 0.004 & 0.008 & 0.290 & 1 \\
\hline$A_{5}$ & 0.011 & 0.004 & 0.011 & 0.004 & 0.006 & 0.010 & 0.004 & 0.008 & 0.057 & 6 \\
\hline$A_{6}$ & 0.011 & 0.004 & 0.098 & 0.004 & 0.006 & 0.010 & 0.004 & 0.073 & 0.209 & 3 \\
\hline
\end{tabular}

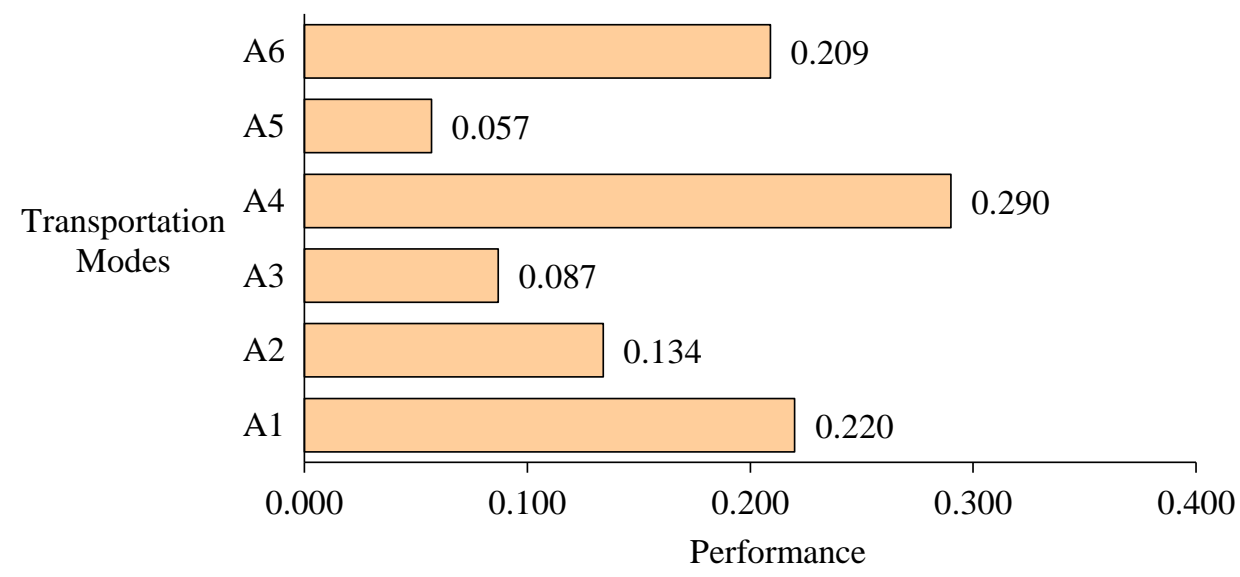

Figure 1. Overall Performance of the Transportation Modes

The values in Table 7 and the bars in Figure 1 show that $A_{4}$ (Taxi) is the best mode of transportation in order to meet the personalised preferences.

\section{Comparative Analysis and Model Validation}

In order to test the validity of the proposed model, we have applied it to an existing case study as given by [9] and a comparative analysis is carried out with the established MCDM technique. For the given case study, AFS theory is used to find the best fuzzy description of decision alternatives (companies $\mathrm{X}, \mathrm{Y}$ and $\mathrm{Z}$ ) and the final ranking are obtained using SAW approach.

The results (Table 8) obtained by the proposed model and that of established fuzzy MCDM approach are same that ensure the acceptability and validity of the developed decision support model. 
Table 8. Comparative Study of Results

\begin{tabular}{|l|c|c|c|c|}
\hline \multirow{2}{*}{ Alternatives } & \multicolumn{2}{|c|}{ Using Proposed Model } & \multicolumn{2}{c|}{ Using Fuzzy MCDM Framework [9] } \\
\cline { 2 - 5 } & Composite Score $\left(A_{i}^{*}\right)$ & Rank & Closeness Coefficient $\left(C C_{i}\right)$ & Rank \\
\hline Company X & 0.3106 & 2 & 0.6946 & 2 \\
\hline Company Y & 0.5713 & 1 & 0.8189 & 1 \\
\hline Company Z & 0.1185 & 3 & 0.6886 & 3 \\
\hline
\end{tabular}

\section{Conclusion}

This study focuses on development of a hybrid decision support model to solve complex multiple criteria decision problems. The developed model incorporates AHP as a multiple criteria decision-making method to determine priorities among decision criteria, and SAW methodology to obtain the ranking of decision alternatives. The best fuzzy description of each decision alternative is obtained using AFS theory and the performance scores of each alternative are determined using pairwise comparisons over cost or benefit criteria as described in alternatives' best fuzzy description. Finally, the resulting scores (decision matrix) are used to rank the decision alternatives using SAW methodology. The axiomatic fuzzy logic is incorporated into the model to overcome the uncertainty and ambiguity in human knowledge representation. The major advantage of the developed model is that it processes the linguistic values using axiomatic fuzzy logic that overcomes the ambiguity in human decision making process and copes with the inconsistency caused by different types of fuzzy numbers. An application of transportation mode selection has been presented to simulate the better understanding of the proposed methodology. As a result of the study, it is found that the proposed model is practical for ranking alternatives with respect to multiple conflicting criteria.

\section{References}

[1] A. Jahan and K. L. Edwards, "A state-of-the-art survey on the influence of normalization techniques in ranking: Improving the materials selection process in engineering design", Materials and Design, vol. 65, (2015), pp. 335-342.

[2] A. John, Z. Yang, R. Riahi and J. Wang, "Application of a collaborative modelling and strategic fuzzy decision support system for selecting appropriate resilience strategies for seaport operations", Journal of Traffic and Transportation Engineering (English Edition), vol. 1, no. 3, (2014), pp. 159-179.

[3] A. Mardani, A. Jusoh and E. K. Zavadskas, "Fuzzy multiple criteria decision-making techniques and applications - Two decades review from 1994 to 2014", Expert Systems with Applications, vol. 42, no. 8, (2015), pp. 4126-4148.

[4] C. Minal and R. Sekhar, "Mode choice analysis: The data, the models and future ahead", International Journal for Traffic and Transport Engineering, vol. 4, no. 3, (2014), pp. 269-285.

[5] E. Thanassoulis, P. K. Dey, K. Petridis, I. Goniadis and A. C. Georgiou, "Evaluating higher education teaching performance using combined analytic hierarchy process and data envelopment analysis", Journal of the Operational Research Society, vol. 68, no. 4, (2017), pp. 431-445.

[6] E. Turban, J. E. Aronson and T. P. Liang, "Decision Support Systems and Intelligent Systems", PearsonPrentice Hall, New Jersey, (2005)

[7] E. Vafeiadis, "An interdisciplinary study of transport mode choice", Aalborg University, Denmark, (2012).

[8] G. A. Shafabakhsh, M. Hadjihoseinlou and S. A. Taghizadeh, "Selecting the appropriate public transportation system to access the Sari International Airport by fuzzy decision making”, European Transport Research Review, vol. 6, (2014), pp. 277-285.

[9] G. Buyukozkan, O. Feyzioglu and E. Nebol, "Selection of the strategic alliance partner in the logistic value chain", International Journal of Production Economics, vol. 113, no. 1, (2008), pp. 148-158.

[10] K. Mukherjee, "Analytic hierarchy process and technique for order preference by similarity to ideal solution: a bibliometric analysis 'from' past, present and future of AHP and TOPSIS", International Journal of Intelligent Engineering Informatics, vol. 2, no. 2/3, (2014), pp. 96-117.

[11] L. Anojkumar, M. Ilangkumaran and S. M. Hassan, "An integrated hybrid multi-criteria decision making technique for material selection in the sugar industry", International Journal of Multicriteria Decision Making, vol. 6, no. 3, (2016), pp. 247-268. 
[12] M. Kumru and P. Y. Kumru, "Analytic hierarchy process application in selecting the mode of transport for a logistics company", Journal of Advanced Transportation, vol. 48, no. 8, (2014), pp. 974-999.

[13] M. S. Kuo, G. S. Liang and W. C. Huang, "Extensions of the multicriteria analysis with pairwise comparison under a fuzzy environment", International Journal of Approximate Reasoning, vol. 43, no. 3, (2006), pp. 268-285.

[14] R. Sarkhel, N. Das, A. K. Saha and M. Nasipuri, "A multi-objective approach towards cost effective isolated handwritten Bangla character and digit recognition", Pattern Recognition, vol. 58, (2016), pp. 172-189.

[15] S. Jain, P. Aggarwal, P. Kumar, S. Singhal and P. Sharma, "Identifying public preferences using multicriteria decision making for assessing the shift of urban commuters from private to public transport: A case study of Delhi”, Transportation Research Part F: Traffic Psychology and Behaviour, vol. 24, (2014), pp. 60-70.

[16] S. O. Odeyale, O. J. Alamu and E. O. Odeyale, "Performance evaluation and selection of best mode of transportation in Lagos state metropolis", International Journal of Traffic and Transport Engineering, vol. 4, no. 2, (2014), pp. 76-89.

[17] S. P. Singh and P. Singh, "A hybrid decision support model using axiomatic fuzzy set theory in AHP and TOPSIS for multicriteria route selection. Complex and Intelligent Systems", (2018), doi: https://doi.org/10.1007/s40747-018-0067-y.

[18] S. P. Singh, M. K. Chauhan and P. Singh, "Using multicriteria futuristic fuzzy decision hierarchy in SWOT analysis: an application in tourism industry", International Journal of Operations Research and Information Systems, vol. 6, no. 4, (2015), pp. 38-56.

[19] T. L. Saaty, "The Analytic Hierarchy Process", McGraw-Hill, New York, (1980).

[20] V. V. Can, "Estimation of travel mode choice for domestic tourists to Nha Trang using the multinomial probit model", Transportation Research Part A, vol. 49, (2013), pp. 149-159.

[21] X. Liu and W. Pedrycz, "Studies in Fuzziness and Soft Computing: Axiomatic Fuzzy Set Theory and its Applications", Edited J. Kacprzyk, Springer, Germany, (2009), pp. 351-421.

[22] X. Liu, "The fuzzy sets and systems based on AFS structure, EI algebra and EII algebra", Fuzzy Sets and Systems, vol. 95, no. 2, (1998), pp. 179-188.

[23] X. Liu, "The fuzzy theory based on AFS algebras and AFS structure", Journal of Mathematical Analysis and Applications, vol. 217, no. 2, (1998), pp. 459-478.

[24] X. Liu, X. Feng and W. Pedrycz, "Extraction of fuzzy rules from fuzzy decision trees: An axiomatic fuzzy sets (AFS) approach", Data \& Knowledge Engineering, vol. 84, (2013), pp. 1-25.

[25] X. Liu, X. Wang and W. Pedrycz, "Fuzzy clustering with semantic interpretation", Applied Soft Computing, vol. 26, (2015), pp. 21-30.

[26] X. Tian, X. Liu and L. Wang, "An improved PROMETHEE II method based on Axiomatic Fuzzy Sets. Neural Computing and Applications", vol. 25, no. 7-8, (2014), pp. 1675-1683.

[27] Y. Li, X. Liu and Y. Chen, Supplier evaluation and selection using Axiomatic Fuzzy Set and DEA methodology in supply chain management", International Journal of Fuzzy Systems, vol. 14, no. 2, (2012), pp. 215-225.

[28] Z. Li, X. Duan, Q. Zhang, C. Wang, Y. Wang and W. Liu, "Multi-ethnic facial features extraction based on axiomatic fuzzy set theory", Neurocomputing, vol. 242, (2017), pp. 161-177.

\section{Authors}

Sunil Pratap Singh is an Assistant Professor at Bharati Vidyapeeth's Institute of Computer Applications and Management, New Delhi, India. He has completed his $\mathrm{PhD}$ in Computer Science from the Dayalbagh Educational Institute, Dayalbagh, Agra, India. His research interests include decision-making techniques and geographical information system (GIS).

Preetvanti Singh is a Professor in the Faculty of Science, Dayalbagh Educational Institute (Deemed University), Dayalbagh, Agra, India. She received her $\mathrm{PhD}$ in Operations Research from the Dayalbagh Educational Institute, Dayalbagh, Agra, India. Her current research interests include decision support system (DSS), geographical information system (GIS) and optimization techniques. 